\title{
Inhibition of antigen-induced bronchial smooth muscle hyperresponsiveness by lovastatin in mice
}

\author{
Yoshihiko CHIBA ${ }^{1}$, Shunsuke SATO $^{1}$ and Miwa MisAwA ${ }^{1}$ \\ ${ }^{1}$ Department of Pharmacology, School of Pharmacy, Hoshi University, 2-4-41 Ebara, \\ Shinagawa-ku, Tokyo 142-8501, Japan
}

Received May 28, 2008; Accepted June 18, 2008

\begin{abstract}
Statins have been proposed as a novel treatment of respiratory diseases. To determine the beneficial effects of statins on the airway hyperresponsiveness, a characteristic feature of allergic bronchial asthma, the effect of systemic treatment with lovastatin on antigeninduced bronchial smooth muscle hyperresponsiveness was investigated in mice. Male $\mathrm{BALB} / \mathrm{c}$ mice were sensitized and repeatedly challenged with ovalbumin antigen. Animals were also treated with lovastatin $(4 \mathrm{mg} / \mathrm{kg} / \mathrm{day}, i . p$.) once a day prior to and during the antigen inhalation period. The bronchial smooth muscle responsiveness to acetylcholine, but not to high $\mathrm{K}^{+}$-depolarization, was markedly and significantly augmented in the repeatedly antigen challenged mice. The bronchial smooth muscle hyperresponsiveness to acetylcholine induced by antigen exposure was significantly attenuated by the systemic treatment with lovastatin. Thus, lovastatin might have therapeutic potential to ameliorate airway hyperresponsiveness in allergic bronchial asthma.
\end{abstract}

Key words: asthma, airway hyperresponsiveness, bronchial smooth muscle, lovastatin, mouse

\section{Introduction}

Increased airway narrowing in response to nonspecific stimuli, called airway hyperresponsiveness (AHR), is a characteristic feature of allergic bronchial asthma. One of the factors that contribute to the exaggerated airway narrowing in asthmatics is an abnormality of the nature of airway smooth muscle (Martin et al., 2000; Seow et al., 1998). It is thus important for development of asthma therapy to understand changes in the contractile signaling of airway smooth muscle cells associated with the disease. To date, there is increasing evidence that a monomeric GTP-binding protein RhoA and its downstream target Rho-kinase are involved in the agonist-induced $\mathrm{Ca}^{2+}$ sensitization of airway smooth muscle contraction (Chiba et al., 1999; 2005, Ito et al., 2001; Yoshii et al., 1999). Recent studies revealed an augmentation of the RhoA/Rhokinase-mediated $\mathrm{Ca}^{2+}$ sensitization in animal models of allergic bronchial asthma (Chiba $e$ t al.,

Correspondence to: Yoshihiko Chiba, Ph.D., Department of Pharmacology, School of Pharmacy, Hoshi

University, 2-4-41 Ebara, Shinagawa-ku, Tokyo 142-8501, Japan

Phone: 81-3-5498-5786 Fax: 81-3-5498-5787 e-mail: chiba@hoshi.ac.jp 
1999; 2005), and the RhoA/Rho-kinase pathway has now been proposed as a new target for the treatment of AHR in asthma (Gosens et al., 2006).

Statins are a class of cholesterol lowering drugs that decrease mortality from cardiovascular disease (Shepherd et al., 1995) and stroke (Briel et al., 2004). The beneficial effects of statins have been attributed to reduce cholesterol biosynthesis through competitive inhibition of 3hydroxy-3-methylglutaryl coenzyme A (HMG-CoA) reductase, which converts HMG-CoA to Lmevalonate. Statins have pleiotropic effects beyond lowering cholesterol levels, such as antiinflammatory actions and anti-oxidant activity (Kumar and Reynolds, 2005; McKay et al., 2004), and have also been proposed as a novel treatment of respiratory diseases (Hothersall et al., 2006). One of the mechanisms of the pleiotropic effects may result from the depletion of the downstream metabolites farnesylpyrophosphate and geranylgeranylpyrophosphate. These isoprenoid pyrophosphates are precursors required for the posttranscriptional prenylation and functional activation of certain proteins, including RhoA. It is thus likely that statins have an ability to reduce the $\mathrm{Ca}^{2+}$ sensitization of smooth muscle contraction via an inhibition of RhoA function indirectly. Indeed, lovastatin can inhibit translocation of RhoA to plasma membrane, which is modulated by its geranylgeranylation, resulting in an inhibition of RhoA-mediated function in cultured tumor cell lines (Lee et al., 2006; Zhong et al., 2003).

In the present study, the effects of lovastatin on the augmented contraction of bronchial smooth muscle in mice with experimental asthma were investigated to determine the beneficial effects of this agent on the AHR in allergic bronchial asthma.

\section{Methods}

Male BALB/c mice were purchased from the Charles River Japan, Inc. (Kanagawa, Japan) and housed in a pathogen-free facility. All animal experiments were approved by the Animal Care Committee of Hoshi University (Tokyo, Japan).

Preparation of a murine model of allergic bronchial asthma was performed as described previously (Chiba et al., 2004; 2005; 2006). In brief, mice (8 weeks of age) were actively sensitized by intraperitoneal injections of $8 \mu \mathrm{g}$ ovalbumin (OA; Seikagaku Co., Tokyo, Japan) with $2 \mathrm{mg}$ Imject Alum (Pierce Biotechnology, Inc., Rockfold, IL, USA) on days 0 and 5. The sensitized mice were challenged with aerosolized OA-saline solution $(5 \mathrm{mg} / \mathrm{ml})$ for $30 \mathrm{~min}$ on days 12,16 and 20 . A control group of mice received the same immunization procedure but inhaled saline aerosol instead of OA challenge. Animals also received intraperitoneal injection with lovastatin (Toronto Research Chemicals, Inc., Ontario, Canada; $4 \mathrm{mg} / \mathrm{kg} /$ day; dissolved in 50\% DMSO in PBS) (Chiba et al., 2008) or its vehicle once a day for 10 days from Day 11 to Day 20. The treatment was carried out $1 \mathrm{~h}$ before the antigen inhalation in case of the days of the antigen challenge (Days 12, 16 and 20). Twenty-four $h$ after the last OA challenge, mice were sacrificed by exsanguination from abdominal aorta under urethane $(1.6 \mathrm{~g} / \mathrm{kg}$, i.p.; Sigma, St. Louis, MO) anesthesia. The smooth muscle responsiveness of the left main bronchial tissues was measured as previous papers (Chiba et al., 2004; 2005). Epithelium was removed by gently rubbing with sharp-edged tweezers.

All the data are expressed as the mean \pm s.e.mean. Statistical significance of difference was determined by unpaired Student's $t$-test or two-way analysis of variance (ANOVA). 
(A)

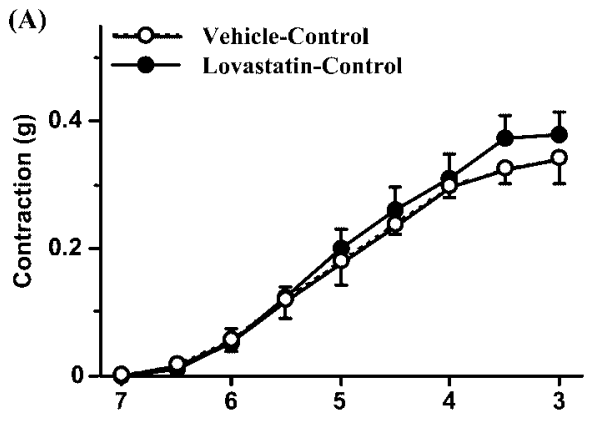

(B)
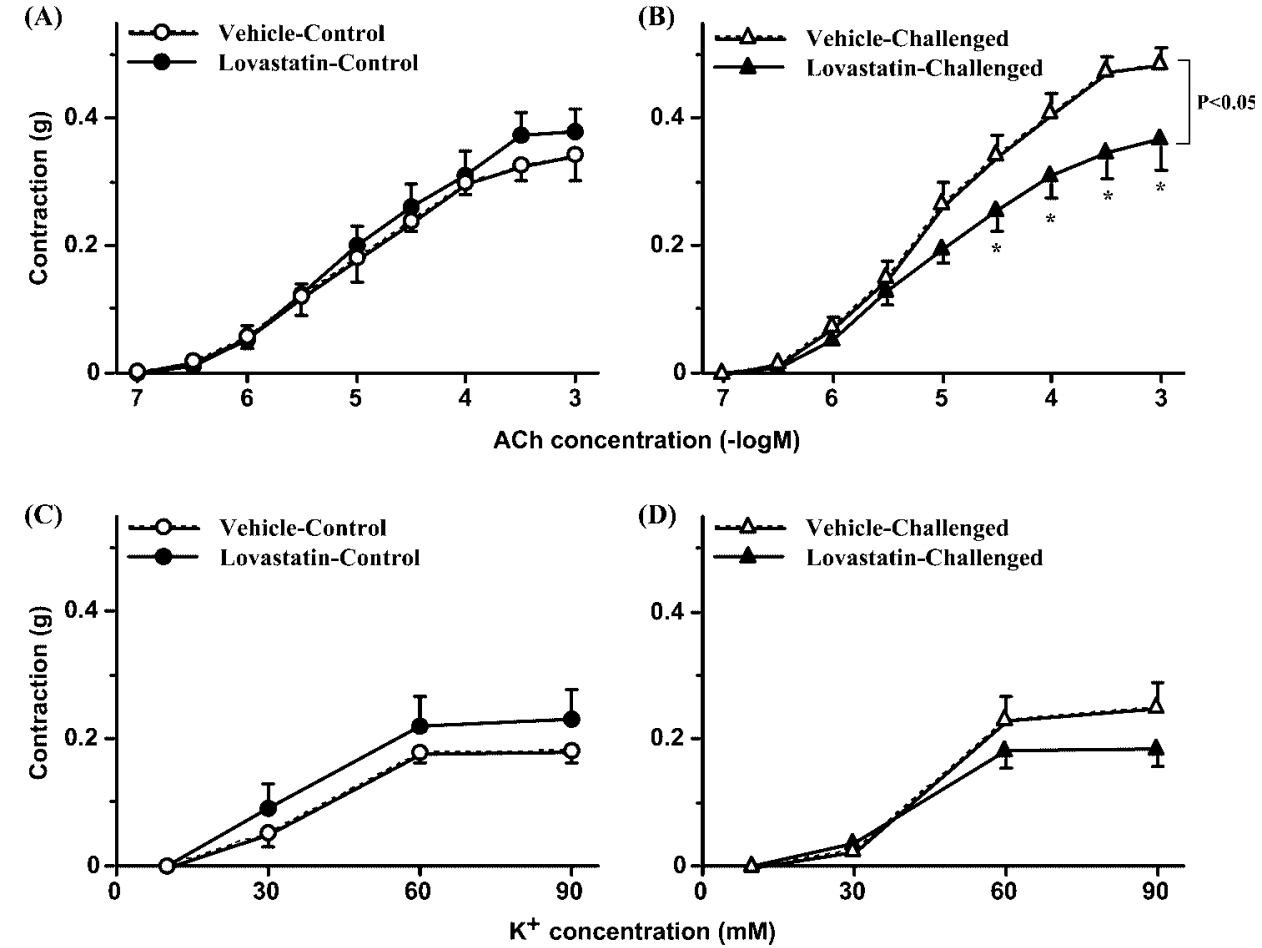

Fig. 1. Effects of systemic treatments with lovastatin on the bronchial smooth muscle hyperresponsiveness induced by repeated antigen exposure. The acetylcholine (ACh; $A$ and $B$ )- and high $\mathrm{K}^{+}$depolarization $(C$ and $D$ )-induced contractions of bronchial smooth muscles isolated from non-sensitized control (Control; $A$ and $C$ ) and repeatedly antigenchallenged (Challenged; $B$ and $D$ ) mice were measured isometrically. Lovastatin (4 $\mathrm{mg}$ / $\mathrm{kg} /$ day) or its vehicle (50\% DMSO in PBS) was also pretreated intraperitoneally for 10 days as described in the METHODS section. Each point represents the mean \pm SEM from 5-6 different animals. Note that the bronchial smooth muscle responsiveness to ACh of the challenged group (Vehicle-Challenged in $B$ ) was significantly augmented as compared with that of the control one (Vehicle-Control in $A ; P<0.05$ by two-way ANOVA), whereas the responsiveness to high $\mathrm{K}^{+}$depolarization was not changed $(C$ and $D$ ). The augmented $\mathrm{ACh}$ responsiveness induced by antigen challenge was significantly inhibited by pretreatment with lovastatin (Vehicle-Challenged $v$ s. Lovastatin-Challenged; $P<0.05$ by two-way ANOVA). ${ }^{*}, P<0.05 v$ s. Lovastatin-Challenged by unpaired Student's $t$-test.

\section{Results and discussion}

Using our well-established mouse model of allergic bronchial asthma (Chiba et al., 2004; 2005; 2006), the effect of systemic treatment with lovastatin on bronchial smooth muscle hyperresponsiveness induced by antigen exposure was determined.

As shown in Figs. 1A and 1B, the contractile response to acetylcholine (ACh) of bronchial smooth muscle from the repeatedly antigen-challenged mice was markedly augmented, i.e., bronchial smooth muscle hyperresponsiveness, as compared to that from the control animals: ACh concentration-response curve was significantly shifted upward by the antigen exposure (Vehicle-Control in Fig. 1A vs. Vehicle-Challenged in Fig. 1B; $P<0.05$ ). The value of maximal 
contraction induced by ACh (Emax; g) of the Vehicle-Challenged group (0.49 \pm 0.03$)$ was significantly greater than that of the Vehicle-Control one $(0.34 \pm 0.04, P<0.05)$, whereas no significant change in the $50 \%$ effective concentration (EC50; - $\log \mathrm{M})$ was observed $(5.07 \pm 0.15$, and $5.14 \pm 0.25$, respectively). On the other hand, no significant change in the contractile response induced by high $\mathrm{K}^{+}$-depolarization (in the presence of $10^{-6} \mathrm{M}$ atropine and $10^{-6} \mathrm{M}$ indomethacin) was observed between the groups (Figs. 1C and 1D).

To determine the effect of lovastatin on the bronchial smooth muscle hyperresponsiveness observed in the repeatedly antigen-challenged mice, animals also received intraperitoneal injection with lovastatin (4 mg/kg/day) or its vehicle (50\% DMSO in PBS) prior to and during the period of repeated antigen exposure. In the non-sensitized normal control animals, the lovastatin treatments had no effect on the contractility of bronchial smooth muscle to ACh (Fig. 1A) or high $\mathrm{K}^{+}$-depolarization (Fig. 1C). However, the bronchial smooth muscle hyperresponsiveness to $\mathrm{ACh}$ observed in the repeatedly antigen-challenged mice was significantly inhibited by the systemic treatments with lovastatin (Fig. $1 \mathrm{~B} ; P<0.05$ ). The bronchial smooth muscle responsiveness to ACh of the antigen-challenged animals completely returned to the control level: no significant difference was observed between the LovastatinChallenged (Fig. 1B) and the Vehicle-Control (Fig. 1A) groups. Similar results have also been obtained in a rat model of allergic bronchial asthma (Chiba et al., 2008), indicating that lovastatin could inhibit bronchial smooth muscle hyperresponsiveness induced by allergen in various species of mammals.

Statins are known to have pleiotropic effects beyond lowering cholesterol levels. It has been reported that simvastatin (McKay et al., 2004) and pravastatin (Yeh and Huang, 2004) showed an anti-inflammatory property in experimental allergic asthma. It is thus possible that the attenuation of bronchial smooth muscle hyperresponsiveness by lovastatin currently observed may result from an inhibition of the antigen-induced airway inflammation. Indeed, airway inflammation determined by total cell counts in bronchoalveolar lavage fluid was also inhibited by lovastatin $\left(4.31 \pm 1.08\right.$ for Vehicle-Challenged and $1.95 \pm 0.38\left(\times 10^{6}\right.$ cells $\left./ \mathrm{ml}\right)$ for LovastatinChallenged groups, repectively; $P<0.01)$. Alternatively, direct effect of lovastatin on the bronchial smooth muscle might also be considerable. Statins inhibit HMG-CoA reductase and thus can reduce the downstream metabolite geranylgeranylpyrophosphate, a precursor required for the posttranslational geranylgeranylation and functional activation of RhoA. RhoA is a critical protein that is involved in the agonist-induced $\mathrm{Ca}^{2+}$ sensitization of smooth muscle contraction. Our previous study revealed an augmentation of acetylcholine-induced translocation of RhoA to the plasma membrane, i.e., an augmented activation of RhoA, in association with the bronchial smooth muscle hyperresponsiveness in a rat model of AHR (Chiba et al., 2001). The augmented RhoA translocation was inhibited by the systemic treatment with lovastatin (Chiba et al., 2008).

In conclusion, the present study demonstrates that lovastatin inhibits the antigen-induced bronchial smooth muscle hyperresponsiveness in mice. Statins, such as lovastatin, may have therapeutic potential to ameliorate AHR in allergic bronchial asthma. 


\section{Acknowledgements}

We thank Mr. Yoshinari Haraguchi for his technical assistance.

\section{References}

Briel, M., Studer, M., Glass, T.R. and Bucher, H.C. (2004). Effects of statins on stroke prevention in patients with and without coronary heart disease: a meta-analysis of randomized controlled trials. Am. J. Med. 117: 596-606.

Chiba, Y., Arima, J., Sakai, H. and Misawa, M. (2008). Lovastatin inhibits bronchial hyperresponsiveness by reducing RhoA signaling in rat allergic asthma. Am. J. Physiol. Lung Cell. Mol. Physiol. 294: L705-L713.

Chiba, Y., Kurotani, R., Kusakabe, T., Miura, T., Link, B.W., Misawa, M. and Kimura, S. (2006). Uteroglobin-related protein 1 expression suppresses allergic airway inflammation in mice. $\mathrm{Am}$. J. Respir. Crit. Care Med. 173: 958-964.

Chiba, Y., Sakai, H. and Misawa, M. (2001). Augmented acetylcholine-induced translocation of RhoA in bronchial smooth muscle from antigen-induced airway hyperresponsive rats. Br. J. Pharmacol. 133: 886-890.

Chiba, Y., Takada, Y., Miyamoto, S., Mitsui-Saito, M., Karaki, H. and Misawa, M. (1999). Augmented acetylcholine-induced, Rho-mediated $\mathrm{Ca}^{2+}$ sensitization of bronchial smooth muscle contraction in antigen-induced airway hyperresponsive rats. Br. J. Pharmacol. 127: 597-600.

Chiba, Y., Ueno, A., Sakai, H. and Misawa, M. (2004). Hyperresponsiveness of bronchial but not tracheal smooth muscle in a murine model of allergic bronchial asthma. Inflamm. Res. 53: 636-642.

Chiba, Y., Ueno, A., Shinozaki, K., Takeyama, H., Nakazawa, S., Sakai, H. and Misawa, M. (2005). Involvement of RhoA-mediated $\mathrm{Ca}^{2+}$ sensitization in antigen-induced bronchial smooth muscle hyperresponsiveness in mice. Respir. Res. 6: Art. No. 4.

Gosens, R., Schaafsma, D., Nelemans, S.A. and Halayko, A.J. (2006). Rho-kinase as a drug target for the treatment of airway hyperresponsiveness in asthma. Mini Rev. Med. Chem. 6: 339-348.

Hothersall, E., McSharry, C. and Thomson, N.C. (2006). Potential therapeutic role for statins in respiratory disease. Thorax 61: 729-734.

Ito, S., Kume, H., Honjo, H., Katoh, H., Kodama, I., Yamaki, K. and Hayashi, H. (2001). Possible involvement of Rho kinase in $\mathrm{Ca}^{2+}$ sensitization and mobilization by $\mathrm{MCh}$ in tracheal smooth muscle. Am. J. Physiol. Lung Cell. Mol. Physiol. 280: L1218-L1224.

Kumar, A.P. and Reynolds, W.F. (2005). Statins downregulate myeloperoxidase gene expression in macrophages. Biochem. Biophys. Res. Commun. 331: 442-451.

Lee, J., Lee, I., Park, C. and Kang, W.K. (2006). Lovastatin-induced RhoA modulation and its effect on senescence in prostate cancer cells. Biochem. Biophys. Res. Commun. 339: 748-754.

Martin, J.G., Duguet, A. and Eidelman, D.H. (2000). The contribution of airway smooth muscle to airway narrowing and airway hyperresponsiveness in disease. Eur. Respir. J. 16: 349-354.

McKay, A., Leung, B.P., McInnes, I.B., Thomson, N.C. and Liew, F.Y. (2004). A novel anti-inflammatory role of simvastatin in a murine model of allergic asthma. J. Immunol. 172: 2903-2908.

Seow, C.Y., Schellenberg, R.R. and Pare, P.D. (1998). Structural and functional changes in the airway smooth muscle of asthmatic subjects. Am. J. Respir. Crit. Care Med. 158: S179-S186.

Shepherd, J., Cobbe, S.M., Ford, I., Isles, C.G., Lorimer, A.R., MacFarlane, P.W., McKillop, J.H. and Packard, C.J. (1995). Prevention of coronary heart disease with pravastatin in men with hypercholesterolemia. West of Scotland Coronary Prevention Study Group. N. Engl. J. Med. 333: 1301-1307.

Yeh, Y.F. and Huang, S.L. (2004). Enhancing effect of dietary cholesterol and inhibitory effect of 
pravastatin on allergic pulmonary inflammation. J. Biomed. Sci. 11: 599-606.

Yoshii, A., Iizuka, K., Dobashi, K., Horie, T., Harada, T., Nakazawa, T. and Mori, M. (1999). Relaxation of contracted rabbit tracheal and human bronchial smooth muscle by Y-27632 through inhibition of $\mathrm{Ca}^{2+}$ sensitization. Am. J. Respir. Cell Mol. Biol. 20: 1190-1200.

Zhong, W.B., Wang, C.Y., Chang, T.C. and Lee, W.S. (2003). Lovastatin induces apoptosis of anaplastic thyroid cancer cells via inhibition of protein geranylgeranylation and de novo protein synthesis. Endocrinology 144: 3852-3859. 